\title{
Correction: Szopinska-Tokov et al. Investigating the Gut Microbiota Composition of Individuals with Attention-Deficit/Hyperactivity Disorder and Association with Symptoms. Microorganisms 2020, 8, 406
}

\author{
Joanna Szopinska-Tokov ${ }^{1,+} \mathbb{D}$, Sarita Dam ${ }^{2,+}$, Jilly Naaijen ${ }^{2} \mathbb{D}$, Prokopis Konstanti ${ }^{3}$, Nanda Rommelse ${ }^{1,4}$, \\ Clara Belzer $^{3}$, Jan Buitelaar 2,4 ${ }^{\text {, Barbara Franke }}{ }^{1,5} \mathbb{D}$, Mirjam Bloemendaal 1,5 ${ }^{(D)}$, Esther Aarts 6 (D) \\ and Alejandro Arias Vasquez ${ }^{1,5, *}$
}

Citation: Szopinska-Tokov, J.; Dam, S.; Naaijen, J.; Konstanti, P.; Rommelse, N.; Belzer, C.; Buitelaar, J.; Franke, B.; Bloemendaal, M.; Aarts, E.; et al. Correction: Szopinska-Tokov et al. Investigating the Gut Microbiota Composition of Individuals with Attention-Deficit/Hyperactivity Disorder and Association with Symptoms. Microorganisms 2020, 8, 406. Microorganisms 2021, 9, 1358. https://doi.org/10.3390/ microorganisms 9071358

Received: 29 April 2021

Accepted: 26 May 2021

Published: 23 June 2021

Publisher's Note: MDPI stays neutral with regard to jurisdictional claims in published maps and institutional affiliations.

Copyright: (c) 2021 by the authors. Licensee MDPI, Basel, Switzerland. This article is an open access article distributed under the terms and conditions of the Creative Commons Attribution (CC BY) license (https:/ / creativecommons.org/licenses/by/ $4.0 /)$.
1 Department of Psychiatry, Radboudumc, Donders Institute for Brain, Cognition and Behaviour, 6525 GA Nijmegen, The Netherlands; Joanna.Szopinska-Tokov@radboudumc.nl (J.S.-T.); Nanda.Lambregts-Rommelse@radboudumc.nl (N.R.); Barbara.Franke@radboudumc.nl (B.F.); Mirjam.Bloemendaal@radboudumc.nl (M.B.)

2 Department of Cognitive Neuroscience, Radboudumc, Donders Institute for Brain, Cognition and Behaviour, 6525 EN Nijmegen, The Netherlands; Sarita.Dam@radboudumc.nl (S.D.); jilly.naaijen@donders.ru.nl (J.N.); Jan.Buitelaar@radboudumc.nl (J.B.)

3 Laboratory of Microbiology, Wageningen University, 6708 WE Wageningen, The Netherlands; prokopis.konstanti@wur.nl (P.K.); clara.belzer@wur.nl (C.B.)

4 Karakter Child and Adolescent Psychiatry University Center, 6525 GC Nijmegen, The Netherlands

5 Department of Human Genetics, Radboudumc, Donders Institute for Brain, Cognition and Behaviour, 6525 GA Nijmegen, The Netherlands

6 Centre for Cognitive Neuroimaging, Donders Institute for Brain, Cognition and Behaviour, Radboud University, 6525 EN Nijmegen, The Netherlands; esther.aarts@donders.ru.nl

* Correspondence: Alejandro.AriasVasquez@radboudumc.nl; Tel.: +31-(0)-24-3613970

+ Authors contributed equally to this work.

The authors wish to make the following correction to this paper [1]:

After the publication of the manuscript, the authors recognized a mismatch in the link between the microbiota sequencing data (from ADHD cases and controls) and their descriptive and behavioral data. Thus, the manuscript had to be reanalyzed and rewritten, resulting in different results and conclusion. The main difference is that the case-control comparison resulted in different bacteria differences. Moreover, we did not find an association (only at trend level) between the microbiome relative abundance and inattention score. The corrected results, discussion, and conclusion, can be found below. Due to the changes the abstract, and material and methods section had to be adjusted as well. The changes are provided below.

The authors would like to apologize for any inconvenience caused to the readers by these changes.

\section{Changes in Abstract}

The results and conclusion in the abstract changed to:

Alpha and Beta-diversity were not different between participants with ADHD and healthy controls. Three genera showed nominal differences $\left(p_{\text {uncorrected }}<0.05\right)$ between both groups (Prevotella_9, Coprococcus_2 and Intestinibacter) and were further tested for their association with ADHD symptom scores (adjusting for age, sex, body mass index, a time delay between feces collection and symptoms assessment, medication use and family relatedness). Our results show that the variation of a genus from the Lachnospiraceae family (Coprococcus_2) showed a trend of being negatively associated with inattention symptoms. Furthermore, we showed that the relative abundance of four genera was reduced by ADHD medication $\left(p_{\text {uncorrected }}<0.05\right)$. Overall, our results may support the 
role of the gut microbiota in the pathophysiology of ADHD. Given the scarcity of studies on the gut microbiota in individuals with ADHD, the current results are an important contribution to this field. More studies are needed into the gut microbiota as part of the pathology of ADHD, especially with a bigger sample size across the lifespan and more detailed information about lifestyle.

\section{Changes in Materials and Methods}

Certain changes had to be applied in the material and methods section. First, for easier maintenance and reproducibility, we used R software instead of SPSS to reanalyze microbiome data. This means that we calculated the alpha-diversity metrics using the $\mathrm{R}$ function microbiome::alpha (version 1.6.0) and the composition analysis using "phyloseq" $\mathrm{R}$ package version 1.28.0. Second, we used the nonparametric Mann-Whitney $U$ test method in order to identify differences in genera between cases and controls. This was visualized by using a boxplot with a summary table representing the number of zeros using "ggpubr" $\mathrm{R}$ package version 0.4.0.999. Third, in the regression analyses, we had to adjust the number of total tests used in FDR to 6 and not 14 tests. Fourth, the new results of the "2.2.9. Correlation Analysis and Multiple Regression with All Selected Genera" are shown in the Supplementary Materials.

\section{Changes in Results}

The data had to be reanalyzed; thus, all the results changed includes all the tables and figures. For easier readability, the whole (corrected) results section is provided below:

\section{Results}

\subsection{Subjects Characteristics}

The general characteristics of the studied sample are presented in Table 1. Mean age, median BMI, percentage of males, and differences in days between fecal collection and ADHD symptoms assessment (diff_days) were similar among the two groups. As expected, mean inattention and hyperactivity/impulsivity scores were statistically different between the ADHD and control groups. Out of the 41 participants with ADHD, 19 were using medication for ADHD.

Table 1. Characteristics of the sample.

\begin{tabular}{|c|c|c|c|c|}
\hline & ADHD & Control & Subthreshold ADHD & $p$-Value ${ }^{\text {a }}$ \\
\hline $\mathrm{N}$ & 41 & 48 & 14 & - \\
\hline Age, mean (SD) & $20.2(4.1)$ & $20.4(3.5)$ & $20.3(3.4)$ & NS \\
\hline Age, range & 13-29 & 13-28 & $14-26$ & - \\
\hline BMI, median (IQR) & $23(20.5-25.5)$ & $22(20-23)$ & $22(20-23)$ & NS \\
\hline BMI, range & $16-31$ & $16-31$ & $20-30$ & - \\
\hline $\mathrm{BMI} \geq 25, \%$ & 29 & 19 & 14 & NS \\
\hline Male, $\%$ & 61 & 50 & 36 & NS \\
\hline Use of ADHD medication, $\mathrm{N}$ & 19 & 0 & 3 & - \\
\hline $\begin{array}{c}\text { Diff_days, median (IQR) } \\
\text { Conners' }\end{array}$ & $17(14-34)$ & $32(13-64)$ & $14.5(10.5-30)$ & NS \\
\hline Inattention, median (IQR) & $64(58-76)$ & $42(38-53)$ & $57(52-64)$ & $<0.001$ \\
\hline $\begin{array}{l}\text { Hyperactivity/Impulsivity, } \\
\text { median (IQR) }\end{array}$ & $56.5(50-64.5)$ & $41(36-49)$ & $57(50-64)$ & $<0.001$ \\
\hline
\end{tabular}

${ }^{a}$ Comparison made for ADHD vs. controls; $t$-test, Mann-Whitney or chi-square test were applied accordingly; one sample had missing value for inattention and hyperactivity/impulsivity scores; four samples had missing value for BMI; four samples were excluded (Figure S1); $\mathrm{NS}$ = not significant; $\mathrm{SD}$ = standard deviation; $\mathrm{IQR}$ = interquartile range; diff_days = represents differences in days between fecal collection and Conner's assessment. 


\subsection{Microbiota Measures}

Within- and between-sample diversity metrics: None of the three alpha-diversity (withinsample diversity) measures showed significant differences between the ADHD and control groups (Figure S2).

Beta-diversity (between-sample diversity), assessed using betadisper [2], showed that the ADHD group had a smaller variation in the gut microbiota composition $(p=0.08$; Figure 1 and Figure S3). PCoA based on weighted UniFrac distance did not show discrimination of microbial composition between the two groups determined by disorder status (ADHD vs. controls) (Figure S3). This was supported by the statistical test-ADONIS, where participants with ADHD and controls samples displayed non-significant separation according to weighted UniFrac distance (variance explained $=0.9 \%, p=0.479, \mathrm{~N}=89$ ). Other variables, such as age, sex, BMI, inattention score (IA), hyperactivity-impulsivity score (HI), and medication, did not show a significant effect on beta-diversity (Table 2).

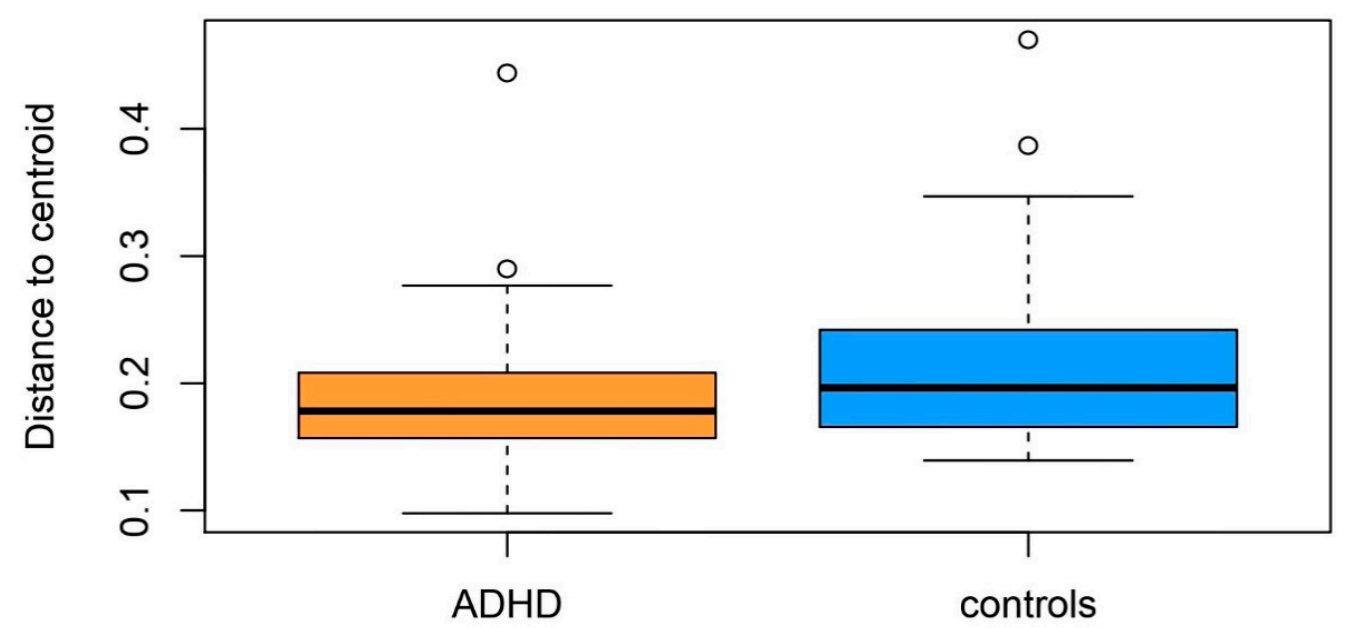

Figure 1. Boxplot of multivariate homogeneity of groups' dispersions (betadisper) of participants with $\mathrm{ADHD}$ and controls. Box plots represent median with whiskers on $\pm 1.5 \mathrm{IQR}$. Pseudo-F =3.051, $p=0.08$.

Table 2. Beta diversity analysis.

\begin{tabular}{ccccc}
\hline Variable & $\mathbf{N}$ & $\mathbf{R}^{\mathbf{2}}$ & Pseudo-F & $p$-Value \\
\hline Disorder status & 89 & 0.009 & 0.79 & 0.479 \\
Age & 103 & 0.005 & 0.55 & 0.727 \\
Sex & 103 & 0.005 & 0.54 & 0.750 \\
BMI & 98 & 0.005 & 0.46 & 0.874 \\
IA & 102 & 0.009 & 0.95 & 0.360 \\
HI & 102 & 0.010 & 1.04 & 0.322 \\
medication & 41 & 0.021 & 0.84 & 0.469 \\
\hline
\end{tabular}

Results of ADONIS on weighted UniFrac dissimilarity matrix including six tests for disorder status, age, sex, BMI, Inattention (IA) and Hyperactivity/Impulsivity (HI) variables; $\mathrm{R}^{2}$ = variance explained, a measure of effect size; Pseudo- $\mathrm{F}=$ indicator of the number of clusters, the larger pseudo-F value, the greater between-group variation than the within-group variation.

\subsubsection{Taxonomic Composition Analysis and Associations with Symptoms}

As expected from [3], a compositional analysis of our samples revealed that Firmicutes, Bacteroidetes, Actinobacteria, Proteobacteria, and Verrucomicrobia, were the most frequent phyla in our data (Table S2). There were no significant differences in the relative abundance of any of these phyla between participants with ADHD and controls (Table S2).

At the genus level, differences in the gut microbiota composition revealed nominal significant case-control differences for three genera $(p<0.05$; Figure 2$)$. Of those, one genus was higher, and two were lower in participants with ADHD compared with control samples. One genus, Coprococcus_2 showed a trend of being negatively associated $(\mathrm{B}=(-3.189)$, 
$p=0.055, Q=0.33$; corrected for multiple testing; Table 3) with inattention scores. We did not find any association between tested genera and hyperactivity/impulsivity scores (before or after correcting for multiple testing; all $p>0.05$ ); therefore, only IA was considered in further analyses.

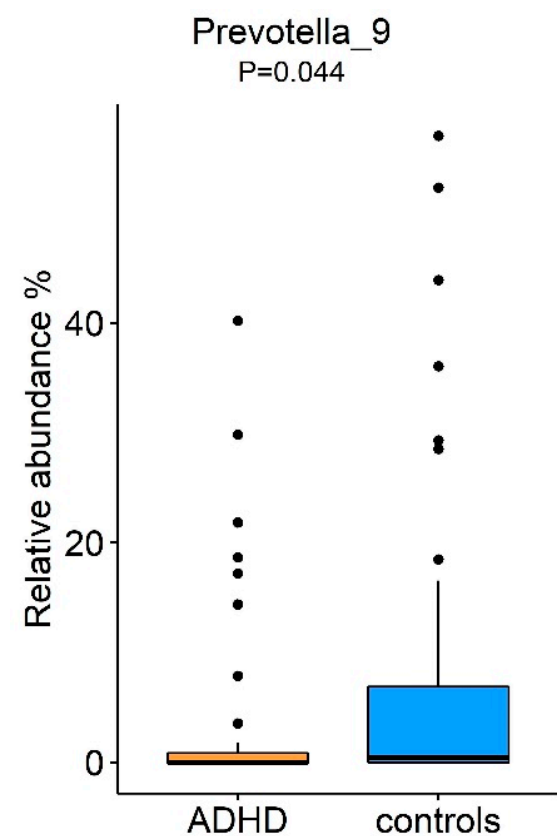

$\begin{array}{rrr}\text { n_zeros } & 26 & 20 \\ \text { n_non-zeros } & 15 & 28\end{array}$

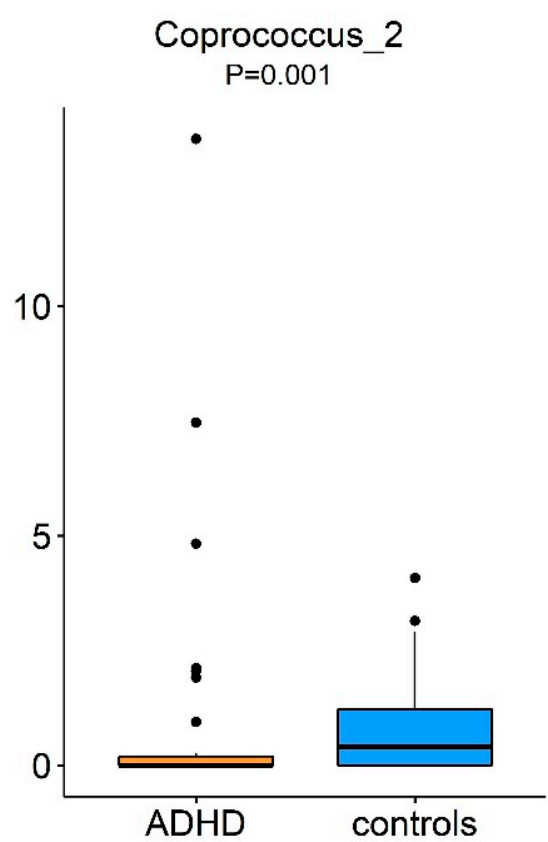

30

11

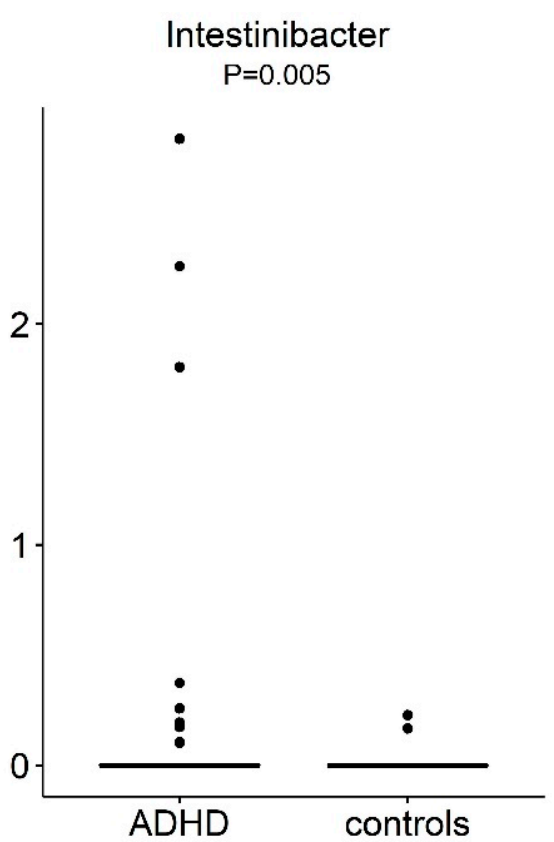

$31 \quad 46$

Figure 2. Comparison of bacterial relative abundance between participants with ADHD and controls. Identification of the bacteria differences was made by the Mann-Whitney test. No outliers were removed since we used a non-parametric test which is less sensitive to the extreme values. Box plots represent median with whiskers on \pm 1.5 IQR. Nominal significant threshold: $p<0.05$.

Table 3. Association of the genera with ADHD symptoms scores.

\begin{tabular}{|c|c|c|c|c|c|c|c|c|}
\hline & \multicolumn{4}{|c|}{ Inattention } & \multicolumn{4}{|c|}{ Hyperactivity/Impulsivity } \\
\hline & $\mathbf{N}$ & $B\left(\right.$ S.E.) ${ }^{a}$ & $95 \%$ CI & $p$-Value & $\mathbf{N}$ & B (S.E.) ${ }^{a}$ & $95 \% \mathrm{CI}$ & $p$-Value \\
\hline Prevotella_9 & 98 & $0.111(0.099)$ & $-0.079-0.306$ & 0.267 & 98 & $0.118(0.096)$ & $-0.065-0.308$ & 0.222 \\
\hline Coprococcus_2 & 95 & $-3.189(1.639)$ & $-6.325-(-0.029)$ & 0.055 & 96 & $-2.331(1.456)$ & $-5.108-0.492$ & 0.113 \\
\hline Intestinibacter & 85 & $191.161(139.654)$ & $-74.119-4.587$ & 0.175 & 94 & $33.829(22.779)$ & $-9.855-77.482$ & 0.141 \\
\hline
\end{tabular}

Linear regression models for the relative abundance of the selected genera (based on the Mann-Whitney U test) with the ADHD symptoms scores (inattention and hyperactivity/impulsivity) measured from participants with ADHD and controls and subthreshold ADHD; ${ }^{a}$ Linear regression model without samples removed based on Cook's distance and Leverage threshold; models adjusted for age, sex, BMI, diff_days and a random factor for family relatedness. There was no significant association after multiple testing correction (FDR); $\mathrm{N}=$ number of samples after the removal of outliers $(\mathrm{N}=98$ means no outliers were removed); $\mathrm{B}=$ coefficient; $\mathrm{S}$.E. = standard error; $\mathrm{CI}=\mathrm{Confidence} \mathrm{Interval}$.

\subsubsection{Effect of Medication on the Regression Results and on Gut Microbiota Composition}

We tested the effect of ADHD medication on the (regression) results by excluding medicated cases $(\mathrm{N}=19)$ from the analysis. We found that medication reduced the beta coefficient from -3.189 to -2.806 in the association between Coprococcus_2 and symptoms of inattention $(\mathrm{B}=(-2.806), p=0.080$ vs. results in Table 3$)$. This reduction can be due to the reduction in sample size $(\mathrm{N}=79$ vs. $\mathrm{N}=95)$.

We performed a post hoc exploratory analysis where we compared the relative abundance of all the genera (total taxa compared $=77$ ) between the medicated $(\mathrm{N}=19)$ vs. non-medicated $(\mathrm{N}=22$ ) individuals with ADHD. We found that four genera (Lactobacillus, Lachnospiraceae_ND3007_group, Ruminococcaceae_g_ and Ruminococcaceae_UCG.014) were 
decreased in medicated ADHD ( $p_{\text {uncorrected }} \leq 0.05$; Figure S4). Regarding the Lactobacillus results, we had to treat them with caution because we only had three non-zero values for medicated cases.

\section{Changes in Discussion}

Due to reanalyzed data and a change in results, the discussion and conclusion were adjusted accordingly. For easier readability, the whole (corrected) discussion section is provided below:

\section{Discussion}

This study aimed to determine the differences in gut microbiota composition between individuals with ADHD and controls and the association between the abundance of the selected genera and the severity of ADHD symptoms (inattention and hyperactivity/impulsivity) accounting for the effects of medication. Our results did not show general differences in microbiota composition (beta-diversity) between the groups. At the taxonomic level, we found nominal (uncorrected significant) differences at the genus level; lower abundance of Prevotella_9 and Coprococcus_2 and higher abundance of Intestinibacter in individuals with ADHD compared to controls. Of these three genera, Coproccocus_2 related most strongly $(p=0.055)$ with ADHD symptoms, specifically Inattention symptoms. Excluding subjects that were using ADHD medication from the regression model slightly reduced the strength of the association. Together this indicates that differences in gut microbiome in this sample of ADHD patients compared with control subjects are subtle.

Our results align with the growing evidence that gut microbiome alterations might be part of the pathology of ADHD [4-8]. The taxa, observed to be nominally different, partly overlap with previous findings. For example, while not the genus showing the largest differences, Aarts et al. also found the genus Coprococcus to be underrepresented in individuals with ADHD [6]. Our lab recently performed a humanization study, in which six randomly selected microbiome samples from the NeuroIMAGE cohort (the cohort studied here) were transplanted into germ-free wild-type mice [9]. Mice colonized with ADHD gut microbiota had increased anxiety-like behavior and showed significantly altered structural and functional brain characteristics. When comparing taxonomy between cases and controls in this humanization approach, again, Coprococcus_2 was found altered. Here, the effect was in the opposing direction; relative abundance was increased in mice colonized with ADHD gut microbiota, wherein the current case-control comparison Coprococcus_2 abundance was higher in controls. Putting aside differences in the directions of effects, the fact that genus Coporococcus_2 surfaces in both case-control comparisons suggest that this is an interesting target for replication in gut microbiota associated with ADHD diagnosis.

Furthermore, an abundance of the genus Prevotella was also found lower in children with ADHD compared with controls [4]. Functionally, Prevotella spp. and some Coprococcus species have been identified as short-chain fatty acids (SCFAs) producers [10], which can be absorbed and used as an energy source by the host [11]. SCFAs producers have been shown to play a potential role in ADHD [12] and autism [13,14] through several of the gut-brain-routes, including their anti-inflammatory effects on the central nervous system.

The only genus with a higher rather than lower relative abundance in cases versus controls, Intestinibacter (belonging to Peptostreptococcaceae), was defined only recently [15]; not much is known about its role in ADHD and human health in general. A potential function may be involved in mucus degradation [16]. Mucus-degrading bacteria are linked to inflammatory bowel disease [17], a comorbid diagnosis seen in neurodevelopmental disorders like ASD [18] or ADHD [19]. Note that the relative abundance of this genus is quite low in both groups, and the statistical difference is based on ten non-zero observations in the ADHD group versus two non-zero observations in the control group. The true abundance of less prevalent bacteria is always more challenging to detect using (16S rRNA) sequencing. The zero observations in the genus Intestinibacter may reflect the true absence 
of a sub-threshold presence of this genus, which should be confirmed and extended in metagenome sequencing.

We did not replicate the differences in the Bifidobacterium genus showing the largest (nominally significant) difference between the ADHD group and controls by Aarts et al., even though this sample overlaps with the current sample (around $40 \%$ ). There are many methodological reasons contributing to a lack of replication between studies, including DNA extraction [20], 16S rRNA gene region [21], bioinformatic pipeline, data processing and analysis [22], sample size and study design. This is a general problem in the microbiome field, limiting replication of important findings. Follow-up studies (keeping comparable methods and including dietary patterns, comorbid conditions (of ADHD) and bacterial transcriptomics, metabolomics and metagenomics) are needed to replicate the current findings and to understand the complex biological mechanisms underlying our results.

A specifically novel contribution in this dataset is the exploratory comparison between medicated $(\mathrm{N}=19)$ and non-medicated individuals $(\mathrm{N}=22)$ with ADHD, which showed four genera with a nominally statistically significant lower relative abundance in medicated individuals. The effects of ADHD medication on gut microbiota are very scarce, especially examined at the genus level and in a sample larger than $\mathrm{n}=$ four unmedicated ADHD patients as was available in Prehn-Kristensen et al., 2018 [4]. However, the size of these medicated versus unmedicated sub-groups is still small, and hence these results should be interpreted with caution and replicated in larger group samples. Generally, psychotropic medication is found, unintendedly, to have anti-bacterial effects and can alter microbial composition [23]. Research into the effects of ADHD medication on the gut-brain axis in ADHD patients is needed, aiming to dissociate between disease-specific and medicationinduced characteristics of the gut microbiota.

This study should be viewed in the context of several strengths and limitations. Our strengths include the use of a sample with high-quality clinical assessment and agematched clinically ascertained controls. The limitations of our study include (i) limited sample size (although it is the largest sample of its kind so far, $\mathrm{N}=98$ ) and (ii) lack of information on lifestyle, dietary patterns (including probiotics) or antibiotic use at the time of feces collection. For the former, we applied two QC steps to deal with a large number of variables (genera), their expected small effects and big interindividual variation of the gut microbiota. First, we applied an uncorrected non-parametric approach (to identify the differences between the two groups, reduce the number of variables and prioritize the selection of candidate taxa). Second, we applied an outlier detection step prior to the regression analysis to reduce the chance of false positives/negatives. For the latter, we were only able to collect information on BMI, and while we acknowledge that this is not enough to account for the effects of diet and lifestyle, it is encouraging to see that there was no BMI difference between the groups. Moreover, we looked for and removed samples with a very low bacterial diversity (high proportion of zeros) by applying a 10\% genus-based frequency cut-off per sample. This step can be used as a proxy for individuals using antibiotics since they would show a smaller bacterial diversity.

In conclusion, we found subtle, uncorrected differences in the microbiota composition between individuals with ADHD and controls, of which alterations in genera Prevotella and Coprococcus have also been found by others. Of the three nominally significant different genera, Coprococcus 2 showed the strongest, though trend level relation with inattention symptoms. Given the scarcity of studies on the gut microbiota in individuals with ADHD, the current results are an important contribution to this field. More studies are needed into the gut microbiota as part of the pathology of ADHD, especially with a bigger sample size across the lifespan and more detailed information about lifestyle.

\section{Change in Supplementary Materials}

The Supplementary Materials were changed accordingly and were included as a separate document. 


\section{Change in Author Names (Add a New One)}

Due to applied changes, we would like to add Mirjam Bloemendaal as a co-author in order to emphasize her significant contribution to this correction; this was approved by all co-authors. She should be recognized for her help in verifying the applied changes, as well as in structuring and writing the correction paper.

Author Contributions: Conceptualization, J.S.-T., B.F. and A.A.V.; methodology, J.S.-T., S.D. and C.B.; software, J.S.-T.; validation, J.S.-T., S.D., M.B. and A.A.V.; formal analysis, J.S.-T.; investigation, J.S.-T., S.D., J.N., E.A. and A.A.V.; resources, J.N., P.K., C.B., J.B., B.F., E.A. and A.A.V.; data curation, J.S.-T.; writing-original draft preparation, J.S.-T. and S.D.; writing-review and editing, J.S.-T., S.D., J.N., P.K., N.R., C.B., J.B., B.F., M.B., E.A., A.A.V.; visualization, J.S.-T.; supervision, A.A.V.; project administration, A.A.V.; funding acquisition, B.F. and A.A.V. All authors have read and agreed to the published version of the manuscript.

Funding: This project has received funding from the European Union's Horizon 2020 research and innovation programme under the Marie Sklodowska-Curie project MiND (grant agreement no. 643051). The funding body had no role in the manuscript. The research leading to these results also received funding from the European Community's Horizon 2020 research and innovation programme through the Eat2beNICE (grant agreement no. 728018), CoCA (grant agreement no. 667302) and CANDY (grant agreement no. 847818) projects, and Netherlands Organization for Scientific Research (NWO) Food Cognition and Behaviour Program in relation to the B3: Brain, Bacteria \& Behaviour project (grant agreement number 057-14-005).

Conflicts of Interest: The authors declare no conflict of interest.

\section{References}

1. Szopinska-Tokov, J.; Dam, S.; Naaijen, J.; Konstanti, P.; Rommelse, N.; Belzer, C.; Buitelaar, J.; Franke, B.; Aarts, E.; Vasquez, A.A. Investigating the Gut Microbiota Composition of Individuals with Attention-Deficit/Hyperactivity Disorder and Association with Symptoms. Microorganisms 2020, 8, 406. [CrossRef]

2. Anderson, M.J.; Ellingsen, K.E.; McArdle, B.H. Multivariate dispersion as a measure of beta diversity. Ecol. Lett. 2006, 9, 683-693. [CrossRef]

3. D'Argenio, V.; Salvatore, F. The role of the gut microbiome in the healthy adult status. Clin. Chim. Acta 2015, 451, 97-102. [CrossRef]

4. Prehn-Kristensen, A.; Zimmermann, A.; Tittmann, L.; Lieb, W.; Schreiber, S.; Baving, L.; Fischer, A. Reduced microbiome alpha diversity in young patients with ADHD. PLoS ONE 2018, 13, e0200728. [CrossRef]

5. Jiang, H.-Y.; Zhou, Y.-Y.; Zhou, G.-L.; Li, Y.-C.; Yuan, J.; Li, X.-H.; Ruan, B. Gut microbiota profiles in treatment-naïve children with attention deficit hyperactivity disorder. Behav. Brain Res. 2018, 347, 408-413. [CrossRef]

6. Aarts, E.; Ederveen, T.; Naaijen, J.; Zwiers, M.P.; Boekhorst, J.; Timmerman, H.M.; Smeekens, S.P.; Netea, M.G.; Buitelaar, J.K.; Franke, B.; et al. Gut microbiome in ADHD and its relation to neural reward anticipation. PLoS ONE 2017, 12, e0183509. [CrossRef] [PubMed]

7. Akram, H. Characterizing a Link between Gut Microbiome and Attention Deficit Hyperactive Disorder. Honors College Research Collection. 2017. Available online: https:/ / digitalcommons.fiu.edu/honors-research/4 (accessed on 5 June 2021).

8. Wang, L.-J.; Yang, C.-Y.; Chou, W.-J.; Lee, M.-J.; Chou, M.-C.; Kuo, H.-C.; Yeh, Y.-M.; Lee, S.-Y.; Huang, L.-H.; Li, S.-C. Gut microbiota and dietary patterns in children with attention-deficit/hyperactivity disorder. Eur. Child. Adolesc. Psychiatry 2019, 29, 287-297. [CrossRef] [PubMed]

9. Tengeler, A.C.; Dam, S.A.; Wiesmann, M.; Naaijen, J.; Van Bodegom, M.; Belzer, C.; Dederen, P.J.; Verweij, V.; Franke, B.; Kozicz, T.; et al. Gut microbiota from persons with attention-deficit/hyperactivity disorder affects the brain in mice. Microbiome 2020, 8, 1-14. [CrossRef] [PubMed]

10. Koh, A.; De Vadder, F.; Kovatcheva-Datchary, P.; Bäckhed, F. From Dietary Fiber to Host Physiology: Short-Chain Fatty Acids as Key Bacterial Metabolites. Cell 2016, 165, 1332-1345. [CrossRef] [PubMed]

11. den Besten, G.; van Eunen, K.; Groen, A.K.; Venema, K.; Reijngoud, D.J.; Bakker, B.M. The role of short-chain fatty acids in the interplay between diet, gut microbiota, and host energy metabolism. J. Lipid Res. 2013, 54, 2325-2340. [CrossRef]

12. Dam, S.A.; Mostert, J.C.; Szopinska-Tokov, J.W.; Bloemendaal, M.; Amato, M.; Arias-Vasquez, A. The Role of the Gut-Brain Axis in Attention-Deficit/Hyperactivity Disorder. Gastroenterol. Clin. N. Am. 2019, 48, 407-431. [CrossRef] [PubMed]

13. Wang, L.; Christophersen, C.T.; Sorich, M.J.; Gerber, J.P.; Angley, M.T.; Conlon, M.A. Elevated Fecal Short Chain Fatty Acid and Ammonia Concentrations in Children with Autism Spectrum Disorder. Dig. Dis. Sci. 2012, 57, 2096-2102. [CrossRef] [PubMed]

14. Abdelli, L.S.; Samsam, A.; Naser, S.A. Propionic Acid Induces Gliosis and Neuro-inflammation through Modulation of PTEN/AKT Pathway in Autism Spectrum Disorder. Sci. Rep. 2019, 9, 1-12. [CrossRef] [PubMed] 
15. Gerritsen, J.; Fuentes, S.; Grievink, W.; Van Niftrik, L.; Tindall, B.J.; Timmerman, H.M.; Rijkers, G.T.; Smidt, H. Characterization of Romboutsia ilealis gen. nov., sp. nov., isolated from the gastro-intestinal tract of a rat, and proposal for the reclassification of five closely related members of the genus Clostridium into the genera Romboutsia gen. nov., Intestinibacter gen. nov., Terrisporobacter gen. nov. and Asaccharospora gen. nov. Int. J. Syst. Evol. Microbiol. 2014, 64, 1600-1616. [CrossRef]

16. Forslund, K.; Hildebrand, F.; Nielsen, T.R.; Falony, G.; Le Chatelier, E.; Sunagawa, S.; Prifti, E.; Vieira-Silva, S.; Gudmundsdottir, V.; Pedersen, H.K.; et al. Disentangling type 2 diabetes and metformin treatment signatures in the human gut microbiota. Nature 2015, 528, 262-266. [CrossRef]

17. Paone, P.; Cani, P.D. Mucus barrier, mucins and gut microbiota: The expected slimy partners? Gut 2020, 69, 2232-2243. [CrossRef] [PubMed]

18. Wasilewska, J.J.; Klukowski, M. Gastrointestinal symptoms and autism spectrum disorder: Links and risks—A possible new overlap syndrome. Pediatr. Health Med. Ther. 2015, 6, 153-166. [CrossRef]

19. Chen, M.H.; Su, T.P.; Chen, Y.S.; Hsu, J.W.; Huang, K.L.; Chang, W.H.; Bai, Y.M. Comorbidity of Allergic and Autoimmune Diseases among Patients with ADHD: A Nationwide Population-Based Study. J. Atten. Disord. 2017, 21, 219-227. [CrossRef]

20. Szopinska, J.W.; Gresse, R.; Van Der Marel, S.; Boekhorst, J.; Lukovac, S.; Van Swam, I.; Franke, B.; Timmerman, H.; Belzer, C.; Vasquez, A.A. Reliability of a participant-friendly fecal collection method for microbiome analyses: A step towards large sample size investigation. BMC Microbiol. 2018, 18, 1-11. [CrossRef]

21. Rintala, A.; Pietilä, S.; Munukka, E.; Eerola, E.; Pursiheimo, J.-P.; Laiho, A.; Pekkala, S.; Huovinen, P. Gut Microbiota Analysis Results Are Highly Dependent on the 16S rRNA Gene Target Region, Whereas the Impact of DNA Extraction Is Minor. J. Biomol. Tech. 2017, 28, 19-30. [CrossRef]

22. Allali, I.; Arnold, J.W.; Roach, J.; Cadenas, M.B.; Butz, N.; Hassan, H.M.; Koci, M.; Ballou, A.; Mendoza, M.; Ali, R.; et al. A comparison of sequencing platforms and bioinformatics pipelines for compositional analysis of the gut microbiome. $B M C$ Microbiol. 2017, 17, 1-16. [CrossRef] [PubMed]

23. Cussotto, S.; Strain, C.R.; Fouhy, F.; Strain, R.G.; Peterson, V.L.; Clarke, G.; Stanton, C.; Dinan, T.G.; Cryan, J.F. Differential effects of psychotropic drugs on microbiome composition and gastrointestinal function. Psychopharmacology 2019, 236, $1671-1685$. [CrossRef] [PubMed] 